\title{
On The Unsteady-Motion Theory of Magnetic Forces for Maglev*
}

by

S. S. Chen, S. Zhu, and Y. Cai

Energy Technology Division

Argonne National Laboratory

9700 S. Cass Avenue

Argonne, IL 60439
The submitted manuscript has been authored by a contractor of the U.S. Government under contract No. W-31-100-ENG-38. Accordingly, the U. S. Government tetains a nonexclusive, royalty-free license to publish or reproduce the published form of this conlribution, or allow others to do so, for U. S. Government
PATMED

FFA 16 1095

OSTI

*This work was performed under the sponsorship of the U.S. Army Corps of Engineers and the Federal Railroad Administration through interagency agreements with the U.S. Department of Energy.

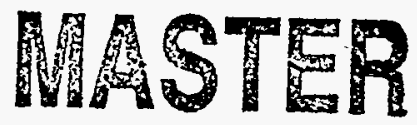




\title{
On The Unsteady-Motion Theory of Magnetic Forces for Maglev
}

by

\author{
S. S. Chen, S. Zhu, and Y. Cai \\ Energy Technology Division \\ Argonne National Laboratory \\ 9700 S. Cass Avenue \\ Argonne, IL 60439
}

\begin{abstract}
Motion-dependent magnetic forces are the key elements in the study of magnetically levitated vehicle (maglev) system dynamics. This paper presents an experimental and analytical study that will enhance our understanding of the role of unsteady-motion-dependent magnetic forces and demonstrate an experimental technique that can be used to measure those unsteady magnetic forces directly. The experimental technique provides a useful tool to measure motion-dependent magnetic forces for the prediction and control of maglev systems.
\end{abstract}

\section{Introduction}

The dynamic response of magnetically levitated vehicle (maglev) systems is very important for the safety, ride quality, guideway design, and cost of these systems. The key elements in the study of maglev system dynamics are magnetic forces. In the past, many studies have been performed to help us understand and 
predict magnetic forces for differing cases. It appears that most maglev-system designs are based on the magnetic forces calculated or measured with the quasisteady-motion theory.

Some limited studies of magnetic forces that consider the effects of unsteady motion have been performed. Magnetic damping was considered by Fujiwara (1980), Iwamoto et al. (1974), Moon (1977), and Yamada et al. (1974). In these investigations, the response of a system supported by or subjected to magnetic forces was measured or analyzed. From the response of the system, the magnetic damping was calculated. This can be considered an indirect method to quantify magnetic forces. Another method is to calculate the magnetic damping by using eddy current losses (Saitoh et al. 1992) or the magnetic forces due to arbitrary motion (Davis and Wilkie 1971). These are approximate solutions that use a direct method that is based on quasisteady motion.

The indirect method has certain limitations. Magnetic coupling, including magnetic damping and stiffness, is difficult to quantify. A direct method will be more useful for identifying each element of the magnetic forces. In addition, it is not clear whether the quasisteady-motion theory is adequate for the development of a stability theory for maglev systems. In some cases, the unsteady magnetic forces are important and the quasisteady-motion theory appears to be inadequate. In developing future maglev systems, at least some scoping calculations on the effects of unsteady magnetic forces must be performed to avoid unexpected failure or excessive operating and maintenance costs.

This paper presents a general approach to the calculation of motion-dependent magnetic forces and an experiment, based on the unsteady-motion theory, to 
measure magnetic forces. The objectives of this study are to: (1) Understand the role of motion-dependent magnetic forces; (2) Provide some theoretical considerations of analytical/numerical methods for calculating unsteady magnetic forces; and (3) Develop and demonstrate an experimental technique that can be used to measure motion-dependent magnetic forces. This study, for the first time, presents a direct method, based on the unsteady-motion theory, to measure motiondependent magnetic forces.

\section{Motion-Dependent Magnetic Forces}

Magnetic forces must be considered in any analysis of vehicle dynamics, guideway structural and fastening design, and prediction of ride comfort (Chen et al. 1992). These forces are considered on the basis of how they relate to vehicle stability. In a vehicle, many magnets or coils are used in the levitation, guidance, and propulsion systems. Without loss of generality, a single magnet or a single coil is used here as an example to demonstrate the role of motion-dependent magnetic forces.

Consider a coil or a magnet with three degrees of freedom, three translations in the propulsion ( $x$ ), guidance (y), and lift (z) directions as shown in Fig. 1. Let $\vec{U}$ be the vector consisting of the three motion components, $u_{1}, u_{2}$, and $u_{3}$, in the three directions $x, y$, and $z$. Velocity and acceleration are given by $\dot{\vec{U}}=\frac{\partial U}{\partial t}$ and $\ddot{\vec{U}}=\frac{\partial^{2} U}{\partial t^{2}}$. The magnetic forces in the three directions can be decomposed into two parts, steady magnetic forces and motion-dependent magnetic forces. 
When the coil or magnet is stationary, the three components of the steady magnetic forces are $f_{10}, f_{20}$, and $f_{30}$. The steady magnetic forces are independent of the motion of the magnet or coil.

When the coil or magnet is moving, the motion-dependent magnetic forces are $f_{1}, f_{2}$, and $f_{3}$. The explicit form of the motion-dependent magnetic forces is not available. It can be written as

$$
f_{i}=\sum_{j=1}^{3}\left(m_{i j} \ddot{u}_{j}+c_{i j} \dot{u}_{j}+k_{i j} u_{j}\right), \quad i=1,2,3
$$

where $\mathrm{m}_{\mathrm{ij}}$, $\mathrm{c}_{\mathrm{ij}}$, and $\mathrm{k}_{\mathrm{ij}}$ are magnetic mass, damping, and stiffness coefficients. Those coefficients are functions of the system parameters and, in general, they are also functions of $u_{j}, \dot{u}_{j}$, and $\ddot{u}_{j}$.

The motion-dependent magnetic forces have been considered from the viewpoint of two theories.

\section{Quasisteady-Motion Theory}

The magnetic forces acting on an oscillating magnet are equal, at any instant in time, to those of the same magnet moving with a constant velocity and with specific clearances equal to the actual instantaneous values. The magnetic forces depend on the deviation from a reference state of speed and clearance, i.e., the motion-dependent magnetic forces depend only on $U$, but not on $\dot{\bar{U}}$ and $\ddot{\bar{U}}$. In this case, $\mathrm{m}_{\mathrm{ij}}$ and $\mathrm{c}_{\mathrm{ij}}$ are zero. Only magnetic stiffnesses are considered. 


\section{Unsteady-Motion Theory}

The magnetic forces acting on an oscillating magnet will depend on $U, \dot{\vec{U}}$, and $\ddot{\vec{U}}$, as shown in Eq. 1. The magnetic force, based on the unsteady-motion theory, can be obtained by calculating or measuring the magnetic force acting on the magnet oscillating in the magnetic field.

\section{Analysis and Experimental Methods}

Two methods can be used to study the motion-dependent magnetic forces, an indirect method and a direct method.

\subsection{Indirect Method}

A magnet or coil that is levitated magnetically can be analyzed theoretically or numerically to predict its response. From the time histories of the magnet or coil displacement, the magnetic damping can be calculated.

In the analysis, various assumptions can be made to make the analysis easier. The motion of a coil or magnet can be calculated numerically on the basis that no mechanical and aerodynamic damping is present. From the time histories of the coil or magnet motion, magnetic damping can be calculated. For example, consider a single-degree-of-freedom magnet levitated on a guideway. From the oscillation frequency and time constant in terms of the decay or amplification amplitude, the magnetic stiffness and damping can be calculated. 
In an experiment, two or three series of tests must be performed to obtain magnetic stiffness and damping.

- Structural stiffness and damping without magnetic field and aerodynamic effect can be measured from the time decay of the structural response to an impact.

- In aerodynamic stiffness and damping, a dummy magnet or coil can be used to measure the combination of stiffness and damping of the structure and aerodynamic effect. From the resultant stiffness and damping, the structural stiffness and damping can be subtracted to obtain aerodynamic stiffness and damping.

- With a magnetic field, the total stiffness and damping will consist of magnetic, aerodynamic, and structural stiffness and damping. Magnetic stiffness and damping can be obtained by subtracting structural and aerodynamic stiffness and damping.

The indirect method requires a series of tests to provide the magnetic stiffness and damping. The system must be clearly quantified before reliable results of magnetic stiffness and damping can be obtained.

\subsection{Direct Method}

\section{Analytical/Numerical Method}

The general procedure to calculate the magnetic forces on the basis of unsteady motion can be summarized as follows: 
Choose a specific case, such as a coil or magnet oscillating over an aluminum conducting sheet (Fig. 1). The coil is levitated at height $\mathrm{z}$ and moving in the $\mathrm{x}$ direction at a speed $v$. The magnetic force components acting on the coil are $f_{1}, f_{2}$, and $f_{3}$ in the three directions, $x, y$, and $z$. The steps described below can be taken to calculate the needed magnetic forces.

(a) Steady Magnetic Force

Set $x=v t, y=0$, and $z=z_{0}$; calculate static magnetic forces, $f_{10}, f_{20}$, and $f_{30}$, which are functions of I (coil current), $h$ (track thickness), and $\sigma$ (track conductivity).

(b) Unsteady Magnetic Forces

Set the condition of the system to one of the following three conditions:

(1) Oscillations in the propulsion direction: $\mathrm{z}=\mathrm{z}_{0}, \mathrm{y}=0, \mathrm{x}=\mathrm{vt}+\mathrm{x}_{1} \cos (\omega \mathrm{t})$.

(2) Oscillations in the guidance direction: $\mathrm{z}=\mathrm{z}_{0}, \mathrm{y}=\mathrm{y}_{1} \cos (\omega \mathrm{t}), \mathrm{x}=\mathrm{vt}$.

(3) Oscillations in the lift direction: $z=z_{0}+z_{1} \cos (\omega t), y=0, x=v t$.

where $x_{1}, y_{1}$, and $z_{1}$ are the oscillation amplitudes of the coil in the propulsion, guidance, and lift directions, respectively, and $\omega$ is the circular frequency of oscillations. 
Under any of the three conditions, the unsteady magnetic force $f_{i}$ can be calculated. For example, when the coil is oscillating in the propulsion direction, the three components are

$$
f_{i}=F_{i}\left(v, z_{0}, x_{1}, I, \omega, t, h, \sigma\right), \quad i=1,2,3
$$

From steady and unsteady magnetic forces, magnetic damping and stiffness matrices can be calculated. The magnetic damping and stiffness for other conditions can be calculated in a similar manner.

It is believed that some motion-dependent magnetic forces for simple geometries can be predicted analytically or numerically. If not, they can be measured experimentally.

\section{Experimental Method}

An experimental method has been developed to measure the unsteady fluid forces acting on a group of tubes in cross flow (Chen 1987; Chen et al. 1993; Tanaka and Takahara 1982). The same technique can be applied to measure magnetic forces.

a. Quasisteady-Motion Theory

The magnetic forces depend on the deviation from a reference state, i.e., the motion-dependent magnetic forces depend only on $u_{j}$, but not $\dot{u}_{j}$ and $\ddot{u}_{j}$, so that 


$$
f_{i}=\sum_{j=1}^{3} k_{i j} u_{j}, \quad i, j=1,2,3
$$

In this case, the magnetic forces are determined uniquely by coil or magnet position. All elements of magnetic stiffness $\mathrm{k}_{\mathrm{ij}}$ can be obtained. To determine $\mathrm{k}_{\mathrm{ij}}$, the magnetic-force component $f_{i}$ is measured as a function of $u_{j}$. Stiffness is given by

$$
\mathbf{k}_{\mathrm{ij}}=\frac{\partial \mathrm{f}_{\mathrm{i}}}{\partial \mathrm{u}_{\mathrm{j}}}
$$

b. Unsteady-Motion Theory

If the displacement component $u_{j}$ is excited, its displacement is given by

$$
\dot{u}_{j}=d_{j} \exp (\sqrt{-1} \omega t)
$$

where $d_{j}$ is the excitation amplitude of the coil or magnet. The motion-dependent magnetic force of the component $f_{i}$ acting on a vehicle is given by

$$
f_{i}=\left[a_{i j} \cos \left(\Psi_{i j}\right)+\sqrt{-1} a_{i j} \sin \left(\Psi_{i j}\right)\right] d_{j} \exp (\sqrt{-1} \omega t)
$$

where $a_{i j}$ is the magnetic force amplitude and $\psi_{\mathrm{ij}}$ is the phase angle between the magnetic force and the vehicle displacement $u_{j}$. These values are measured experimentally.

Using Eqs. 1 and 5, we can also write the motion-dependent magnetic force component as 


$$
f_{i}=\left(-m_{i j} \omega^{2}+\sqrt{-1} \omega c_{i j}+k_{i j}\right) d_{j} \exp (\sqrt{-1} \omega t)
$$

A comparison of Eqs. 6 and 7 and solving for $c_{i j}$ and $k_{\mathrm{ij}}$ yields

$$
\begin{aligned}
& c_{i j}=a_{i j} \sin \left(\psi_{i j}\right) / \omega, \\
& m_{i j}=\left[k_{i j}-a_{i j} \cos \left(\psi_{i j}\right)\right] / \omega^{2} .
\end{aligned}
$$

Based on Eqs. 4 and 8, all motion-dependent magnetic-force matrices can be determined from two experiments: quasisteady-motion and unsteady motion.

If $m_{i j}$ and $c_{i j}$ are of no concern, the experiment that uses quasisteady motion is sufficient to determine $\mathrm{k}_{\mathrm{ij}}$.

\section{Experimental Setup}

The general experimental setup, which includes a rotating drum, shaker, force transducer, and displacement transducer, is shown in Fig. 2. The aluminum wheel is a rotating drum covered with a $14.61-\mathrm{cm}(5-3 / 4-i n$.)-wide, $6.35-\mathrm{mm}$ (1/4-in.)-thick aluminum sheet rolled to a diameter of $26.99 \mathrm{~cm}$ (10 5/8 in.). The rotating speed can vary from 0 to $3500 \mathrm{r} / \mathrm{min}$; therefore, the speed of the wheel is 0 to $50 \mathrm{~m} / \mathrm{s}$. The shaker provides proper excitation force at given frequencies; and the impedance transducer measures the displacement of the supporting bar.

A schematic representation of a force transducer and magnet support is shown in Fig. 3. The magnet is $25.4 \times 50.8 \times 3.18 \mathrm{~mm}$. The magnet is supported by an

aluminum bar $2.54 \mathrm{~cm}$ (1.0 in.) wide, $22.86 \mathrm{~cm}$ (9.0 in.) long (A to E), and $1.27 \mathrm{~cm}$ (0.5 in.) thick. The supporting bar is connected to the shaker at one end and to the 
magnet at the other. At one end it is machined to a thickness of $\approx 4.78 \mathrm{~mm}$ ( $0.188 \mathrm{in}$.). The width is reduced to $1.27 \mathrm{~cm}$. One set of strain gauges is placed on the surface of the smaller section. The strain gauges measure the force components in the lift direction with a sensitivity of $\approx 1 \mathrm{v}$ for $120 \mathrm{~g}$ of force acting on the middle of the magnet.

The supporting bar with the magnet fixed to the shaker at the upper end is fairly rigid with a natural frequency of $>100 \mathrm{~Hz}$. In the tests, the frequencies of interest are $<20 \mathrm{~Hz}$. Therefore, the response of the supporting bar will not affect the measurement of magnetic forces.

The force transducer is calibrated by both static and dynamic methods. In the static method, the supporting bar is attached to the shaker at one end and a given force is applied to the middle of the active length (DE). Because the supporting bar is an elastic beam, the applied force can be correlated with the displacement measured by the displacement transducer and the change of the clearance between the drum and magnet. Therefore, once the displacement is measured by the displacement transducer, the steady magnetic force and the clearance between the magnet and drum can be calculated. The steady magnetic lift force $f(g)$ is given by $\mathrm{f}=403.9 \mathrm{~d}$, where $\mathrm{d}(\mathrm{mm})$ is the displacement measured by the displacement transducer. The change of the clearance $G(\mathrm{~mm})$ between the magnet and drum is given by $G=0.004117 \mathrm{f}$. This means that for a static force applied to the magnet, the clearance will be increased by $G$.

In the dynamic method, the force transducer is excited at a given frequency and amplitude. Then, the inertia forces due to the sinusoidal oscillations are used to determine the calibration constant. 
All calibration constants used in the measurement of motion-dependent magnetic forces are determined by the dynamic method. The inertia force is given by

$$
f_{j}=m \omega^{2} d \cos (\omega t)
$$

where $\omega$ is the oscillation frequency $(\mathrm{rad} / \mathrm{sec}), \mathrm{d}$ is oscillation peak amplitude, and $\mathrm{m}$ is the mass of the active element, which is shown in Fig. $3 \mathrm{~b}$. The active element includes the lower portion of the supporting bar (from the middle of the strain gauge to the lower end), magnet, and aluminum or copper plate. In testing, the inertia force $f_{j}$ is measured for a given excitation $\omega$ and vibration amplitude $d$. In the calibration, $\omega$ varies from $\approx 2$ to $8 \mathrm{~Hz}$ and d varies from 0.8 to $1.1 \mathrm{~mm}$. From Eq. 9, the ratio of $f_{j} / d$ is proportional to $\omega^{2}$; the actual power from experimental data is very close to 2.0 .

\section{Test Procedures and Data Analysis}

The shaker provides a sinusoidal displacement with a frequency varying from $\approx 2$ to $15 \mathrm{~Hz}$. Displacement and force signals can be filtered first to eliminate lowand high-frequency noises. These signals are analyzed to obtain the oscillation displacement of the supporting bar, the magnitudes of the forces acting on the magnet, and the phase between the motion-dependent magnetic force and the displacement of the supporting bar.

Three series of tests were performed, as shown in Fig. $3 \mathrm{~b}$ and Table 1. 
Table 1. Summary of test cases

\begin{tabular}{cccc}
\hline Test Cases & Material & Clearance, $\mathrm{mm}^{\mathrm{a}}$ & Total Mass, $\mathrm{g}^{\mathrm{b}}$ \\
\hline A.1 & Magnet Only & 10.5 & 96.2 \\
A.2 & Magnet Only & 7.5 & 96.2 \\
& & & \\
A.3 & Magnet Only & $6.5,8.5,10.5$, & 96.2 \\
B & $\begin{array}{l}\text { Magnet and } \\
\text { Aluminum Plate }\end{array}$ & 4.5 & 133 \\
C.1 & $\begin{array}{l}\text { Magnet and } \\
\text { Copper Plate }\end{array}$ & 7.7 & 186.5 \\
& $\begin{array}{l}\text { Magnet and } \\
\text { C. }\end{array}$ & 3 & 226 \\
& Copper Plate & & \\
\hline
\end{tabular}

aGap between the aluminum sheet of the drum and the magnet, aluminum plate, or copper plate.

bMass of active element shown in Fig. 3b, including supporting aluminum bar below middle of the strain gauge, magnet, and aluminum or copper plate. 
A. Magnet: Only the magnet is attached to the force transducer. The clearance between the magnet and rotating drum can be set at different values.

B. Magnet and Aluminum Plate: An aluminum plate $4.75 \mathrm{~mm}$ thick, $7.62 \mathrm{~cm}$ long, and $5.08 \mathrm{~cm}$ wide is attached to the magnet. The clearance between the magnet and rotating drum was set at $10.5 \mathrm{~mm}$.

C. Magnet and Copper Plate: A copper plate $1.55 \mathrm{~mm}$ thick, $10.16 \mathrm{~cm}$ long, and $7.62 \mathrm{~cm}$ wide is attached to the magnet. The clearance between the magnet and rotating drum was set at $10.5 \mathrm{~mm}$, while the copper plate can be placed in the gap with different clearance with respect to the rotating drum.

The magnet may be replaced by a dummy steel plate with the same size and same weight as the magnet. With the dummy plate, the dynamic characteristics of the supporting structure, as well as the aerodynamics, can be characterized. In all of the experiments, two tests must be performed: one with the magnet and the other with the dummy steel plate.

In the three series of tests, the clearance $G$ may vary. The test cases are summarized in Table 1. Test Case A is the reference case, whereas the others are performed to enhance our understanding of the effects of the additional plate on the motion-dependent magnetic forces. 


\section{Test Results}

The steady magnetic force depends on the rotating speed of the drum and the clearance. Tests were performed for clearances from $\approx 6.5$ to $14.5 \mathrm{~mm}$ for the different cases.

The measured dynamic force is given as follows:

$$
f=-\frac{\mathrm{md}^{2} \mathrm{u}}{\mathrm{dt}^{2}}-\mathrm{C} \frac{\mathrm{du}}{\mathrm{dt}}-\mathrm{Ku}
$$

where $u$ is the displacement, $m$ is the mass of the active element, denoted by total mass in Table 1 , and $\mathrm{C}$ and $\mathrm{K}$ are damping coefficient and stiffness to be determined, respectively. Note that $m$ should also include magnetic mass, as shown in Eq. 5. However, because the oscillation frequency of maglev is fairly low, the magnetic mass will be very small and is negligible (Iwamoto et al. 1974).

From the time signals of the force transducer and displacement, the RMS values of the displacement and force are $d_{0}$ and $f_{0}$, respectively, and the phase angle between the two is $\phi$ which can be obtained from the correlation between the two time histories of magnetic force and displacement. $\mathrm{C}$ and $\mathrm{K}$ are given by

$$
\begin{aligned}
& \mathrm{C}=\mathrm{f}_{0} \sin (\phi) / \omega \mathrm{u}_{0} \\
& \mathrm{~K}=\mathrm{m} \omega^{2}-\left(\mathrm{f}_{0} / \mathrm{u}_{0}\right) \cos (\phi) .
\end{aligned}
$$

For a given magnet or coil on a sheet guideway, the magnitude of the force depends on excitation amplitude, frequency, and speed. The frequency of magnetic 
force is the same as the excitation frequency; however, the magnetic force is not in phase with the displacement. Depending on the phase between the magnet displacement and magnetic forces, the magnetic forces may be an excitation mechanism or energy dissipation mechanism. A damping force will contribute to energy dissipation, which will reduce maglev response, whereas an excitation force will contribute to negative damping, which may result in dynamic instability.

The detailed results of the tests can be found in a recent report (Chen et al. 1994). The main results will be summarized in this paper.

Figure 4 shows typical experimental data, the steady magnetic force, RMS displacement, RMS magnetic force, and phase angle for Test A.1 with the magnet. From Fig. 4 and Eq. 11, the total stiffness and damping can be calculated. In this test, the stiffness and damping consist of aerodynamic and magnetic effect. The same test was also conducted by replacing the magnet with a dummy; from those results, aerodynamic stiffness and damping can be calculated. In all tests, aerodynamic damping and stiffness are very small. By subtracting the aerodynamic stiffness and damping from the total stiffness and damping will give magnetic stiffness and damping. All magnetic stiffness and damping were obtained by this procedure. Figures 5 and 6 show the magnetic stiffness and damping for Tests A.1, A.2, B, C.1, an C.2 as a function of speed for two oscillating frequencies, 2 and $6 \mathrm{~Hz}$. Figure 7 presents the results for Test A.3 to show the effect of the clearance and excitation frequency, which were performed at $50 \mathrm{~m} / \mathrm{s}$. Figure 8 show magnetic stiffness and damping as a function of excitation amplitude at $33.6 \mathrm{~m} / \mathrm{s}$ at two frequencies, 6 and $10 \mathrm{~Hz}$.

The following general characteristics were noted: 
- The steady magnetic force increases with speed (Fig. 4). The results compare reasonably well with previous measurements (Cai et al. 1992).

- The phase angle between the force and displacement change drastically at the characteristic speed (about $7 \mathrm{~m} / \mathrm{s}$ ), which is equal to $2 / \mu \sigma \mathrm{h}[\mu$ is the permeability, $\sigma$ is the aluminum sheet conductivity, and $h$ is the smaller of aluminum sheet thickness and skin thickness (Sinha 1987)]; therefore the damping coefficient changes sign when the speed is increased to the characteristics speed. At zero speed, the magnetic damping is always positive and magnetic stiffness is zero. When the speed is lower than the characteristic speed, magnetic damping is positive. As the speed increases, it becomes negative (Figs. 5 and 6). At high speed, which is much greater than the characteristic speed, the damping and stiffness are almost independent of the speed.

- The absolute magnitudes of magnetic stiffness and damping increase with reducing clearance. This is noted by comparing the results of Test A.1 and A.2 and from Fig. 5.

- Magnetic damping and stiffness do not change significantly with excitation frequency. This can be seen from Figs. 5 and 6 for different tests at 2 and $6 \mathrm{~Hz}$, and Fig. 7 for variation of frequency from 2 to $6 \mathrm{~Hz}$. This implies that the data measured at a specific frequency can be used for other frequencies without much error.

- In the amplitude range tested, the effect of excitation amplitude is very small. Therefore, for small oscillations, the magnetic damping and stiffness can be 
considered as independent of oscillation amplitude. If the motion is very large, nonlinear effects are expected to become important.

- The effect of the additional aluminum plate or copper plate can be examined by comparing the results of Tests B, C.1, and C.2 with Test A.1. In the parameter range tested, additional plate attached to the magnet does not change the magnetic damping and stiffness significantly. Other geometrical shapes or different materials at various temperatures will be needed to generate sufficient passive damping for maglev.

- With a fixed clearance between the magnet and rotating drum, the magnetic damping due to the copper plate placed between the magnet and drum increases with the decrease of the clearance between the plate and drum (Fig. 6).

- The general magnetic damping characteristics are similar to the train and track loop (Iwamoto et al. 1974). Magnetic damping is negative once the speed is larger than the characteristic speed.

\section{Applications}

In this study, we measured the lift force due to the oscillation in the lift direction. The magnetic damping and stiffness are due to the motion of itself; in general, they are called self-induced damping and stiffness. In reality, once the magnet is given a motion in one direction, it will also induce magnetic forces in other directions. Specifically, if the magnet is excited in the lift direction, three components of the induced forces in the lift, drag, and guidance directions will be induced. In experiments, similar designs of the support and force transducers can 
be developed to measure the three force components that are attributable to an excitation in a direction. From the time histories of these three components of magnetic forces, as well as the phases between the force components and displacement, the magnetic damping and stiffness in the three directions can be calculated. The magnetic damping and stiffness in one direction due to motion in another direction are called coupled magnetic damping and stiffness. For example, for a magnet with the force in three directions, the resulting magnetic damping and stiffness are the same as those given in Eq. 1.

The method presented in this paper is applicable to general cases. If the magnetic damping and stiffness cannot be calculated, the direct method presented here will be useful. In some cases in which the coupled damping and stiffness are not important, the indirect method may be used to measure the self-induced damping and stiffness. When the coupling effect is important, it will be difficult to identify all of the elements of magnetic damping and stiffness matrices from the results of the indirect method.

The experimental data presented earlier can be applied to the dynamics of a maglev vehicle. Once the magnetic damping and stiffness are known, the response and stability of maglev systems can be predicted. Because we measured only the lift force in this study, only the oscillation in the lift direction is considered.

Consider a maglev vehicle, with the mass $m$, rising in a guideway. The applicable equation is

$$
m \frac{d^{2} u}{d t^{2}}+\left(C_{s}+C\right) \frac{d u}{d t}+K u=q(t)
$$


where $\mathrm{m}$ is the total mass of the vehicle; $\mathrm{C}_{\mathrm{s}}$ is structural damping, including aerodynamic damping; $C$ is magnetic damping; $K$ is magnetic stiffness; and $q(t)$ is external excitation.

The natural frequency and modal damping ratio are $f=\frac{1}{2 \pi} \sqrt{\frac{K}{m}}$ and $\zeta=\frac{\mathrm{C}_{\mathrm{S}}+\mathrm{C}}{2 \sqrt{\mathrm{Km}}}$. For a given vehicle, $\mathrm{C}$ and $\mathrm{K}$ depend on clearance and speed; both natural frequency and modal damping will depend on clearance and speed. When the damping value is zero, i.e., $\zeta=0$, or $\mathrm{C}_{\mathrm{s}}+\mathrm{C}=0$, instability will occur.

Consider a specific case. A vehicle with mass equal to $150 \mathrm{~g}$ travels at a speed of $50 \mathrm{~m} / \mathrm{s}$. The magnetic damping and stiffness are given in Fig. 7. The natural frequency and modal damping due to magnetic forces can be calculated assuming $\mathbf{C}_{\boldsymbol{s}}$ $=0$. The results are given in Fig. 9. As the clearance increases, the natural frequency and negative magnetic damping decrease.

To predict the dynamic response of a maglev system, magnetic damping and stiffness matrices must be quantified. Using the direct method, either analytically or experimentally, to characterize magnetic damping and stiffness, including selfinduced and coupling terms, we can predict a detailed analysis of maglev stability and response.

\section{Closing Remarks}

Motion-dependent magnetic forces are the controlling elements in the prediction of dynamic response and stability of maglev systems. At this time, very limited studies have been published and many stability issues remain unresolved. 
An integrated analytical/experimental study has been initiated at Argonne National Laboratory (ANL) to develop an experimental method and computational tools for the study of motion-dependent magnetic forces. This paper presents a general analytical approach and an experimental technique to measure the motiondependent magnetic forces by a direct method. The method is applicable to general cases and will provide the necessary information on the magnetic forces of maglev systems and resolve the issues of maglev stability.

Application of the experimental method presented in this paper can

- Provide the key elements of magnetic forces for stability analysis of maglev systems. Once the magnetic damping and stiffness coefficients for the coil and magnet are known, these coefficients can be applied to maglev systems.

- Characterize the general characteristics of magnetic damping and magnetic stiffness. It might be possible to identify some nondimensional parameters that can be used to determine the role of quasisteady- and unsteady-motion theories.

- Provide the answer to the question: what are the dominant elements that cause instability in maglev systems? Magnetic damping or magnetic stiffness? Selfinduced magnetic damping or coupling of magnetic stiffness?

- Enable the development of a more efficient method of calculating magnetic damping and stiffness coefficients by enhancing our understanding of the general characteristics of magnetic damping and stiffness. 
- Enable us to perform a complete stability analysis of maglev systems by exploring motion-dependent magnetic forces. It will be possible to resolve a series of unsettled issues on maglev stability.

This study represents the first step in the development of magnetic damping and stiffness for maglev. The experimental technique that uses the direct method will provide the necessary elements of magnetic forces. Additional investigation can be performed: (1) conducting plates or coils will be inserted between the magnet and drum to study the role of passive damping; and (2) a magnet will be excited in one direction and the forces in several directions will be measured simultaneously. The results from these studies will improve our understanding of the dynamics of maglev systems.

\section{Acknowledgments}

This work was performed under the sponsorship of the U.S. Army Corps of Engineers and the Federal Railroad Administration through interagency agreements with the U.S. Department of Energy.

The authors appreciate the guidance and interest of Drs. Donald M. Rote and Howard T. Coffey, Manager and Deputy Manager of the ANL Maglev Program. Additional thanks are extended to other members of the ANL Maglev Group, Drs. J. $\mathrm{He}$, J. Hull, T. Mulcahy, and Z. Wang, for many discussions of this work. 


\section{References}

Cai, Y., Chen, S. S., Mulcahy, T. M., and Rote, D. M., 1992, Dynamic Stability of Maglev Systems, Argonne National Laboratory Report ANL-92/21.

Chen, S. S., 1987, Flow-Induced Vibration of Circular Cylindrical Structures, Hemisphere Publishing Corp., New York.

Chen, S. S., Rote, D. M., and Coffey, H. T., 1992, A Review of Vehicle/Guideway Interactions in Maglev Systems, ASME Publication Vol. 231, Fluid-Structure Interaction, Transient Thermal-Hydraulics, and Structural Mechanics, pp. 81-95.

Chen, S. S., Zhu, S., and Jendrzejczyk, J. A., 1993, Motion-Dependent Fluid Forces Acting on Tube Arrays in Crossflow, Argonne National Laboratory Report ANL-93/15.

Chen, S. S., Zhu, S., Cai, Y., 1993, On the Unsteady-Motion Theory of Magnetic Forces for Maglev,"Argonne National Laboratory Report ANL-93/39.

Davis, L. C., and Wilkie, D. F., 1971, Analysis of Motion of Magnetic Levitation Systems: Implications for High-Speed Vehicles, J. Appl. Phys., Vol. 42, No. 12, pp. 4779-4793.

Fujiwara, S., 1980, Damping Characteristics of the Repulsive Magnetic Levitation Vehicle, Japanese Railway Technical Research Institute, Quarterly Reports, Vol. 21, No. 1, pp. 49-52. 
Iwamoto, M., Yamada, T., and Ohno, E., 1974, Magnetic Damping Force in Electrically Suspended Trains, IEEE Trans. Magn., Vol. Mag-10, No. 3, pp. 458-461.

Moon, F. C., 1977, Vibration Problems in Magnetic Levitation and Propulsion, Chapter 6 of Transportation without Wheels by E. R. Laithwaite, Paul Elek (Scientific Books) Ltd., London, pp. 123-161.

Saitoh, T., Maki, N., Kobayashi, T., Shibata, M., and Takizawa, T., 1992, Electromagnetic Force and Eddy Current Loss in Dynamic Behavior of a Superconducting Magnetically Levitated Vehicle, Presented at the Applied Superconductivity Conference, Chicago, August 26, 1992, Paper LKC-1.

Sinha, P. K., 1987, Electromagnetic Suspension - Dynamics and Control, Peter Pergrinus Ltd., England.

Tanaka, H., and Takahara, S., 1982, Fluid Elastic Vibration of Tube Array in Cross Flow, J. Sound Vibration, Vol. 77, pp. 19-37.

Yamada, T., Iwamoto, M., and Ito, T., 1974, Magnetic Damping Force in Inductive Magnetic Levitation System for High-Speed Trains, Elect. Eng. Jpn, Vol. 94, No. 1, pp. 49-54. 


\section{Figure Captions}

Fig. 1. Coil moving over an aluminum conducting sheet

Fig. 2. Experimental setup

Fig. 3. Force transducer and magnet support; (a) overall view; (b) active elements for three test cases

Fig. 4. Experimental data for Test A.1 with magnet, $2 \mathrm{~Hz}$

Fig. 5. Magnetic damping and stiffness, Tests A.1 and A.2

Fig. 6. Magnetic damping and stiffness, Tests B, C.1, and C.2

Fig. 7. (a) Magnetic damping and (b) magnetic stiffness as a function of frequency for various clearances, Test A.3, $v=50 \mathrm{~m} / \mathrm{s}$

Fig. 8. (a) Magnetic damping and (b) magnetic stiffness as a function of excitation amplitude, Test A.1, $33.6 \mathrm{~m} / \mathrm{s}$

Fig. 9. Natural frequency and magnetic modal damping ratio, $v=50 \mathrm{~m} / \mathrm{s}$ 

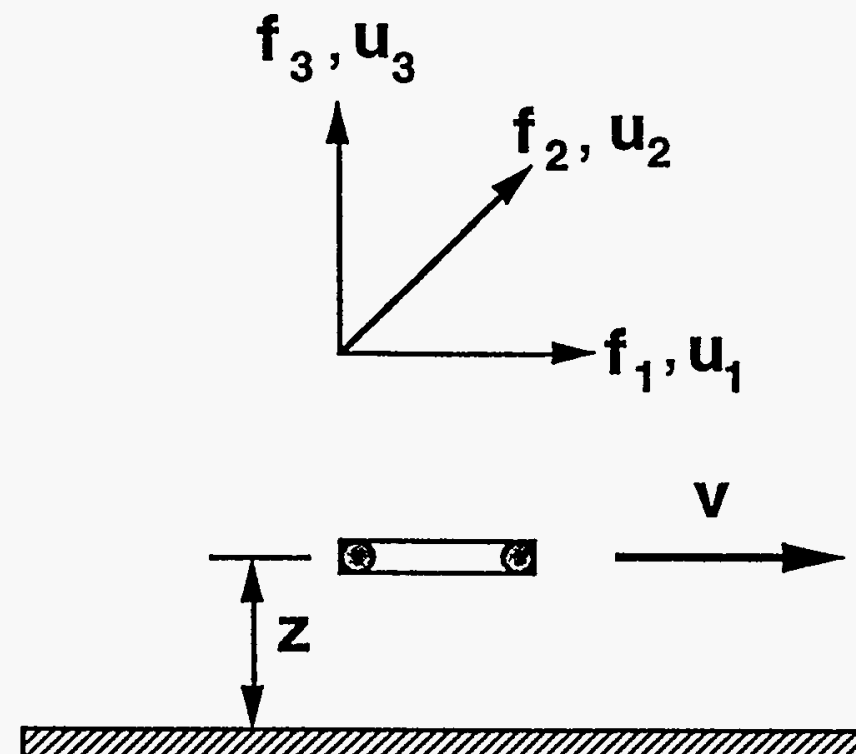

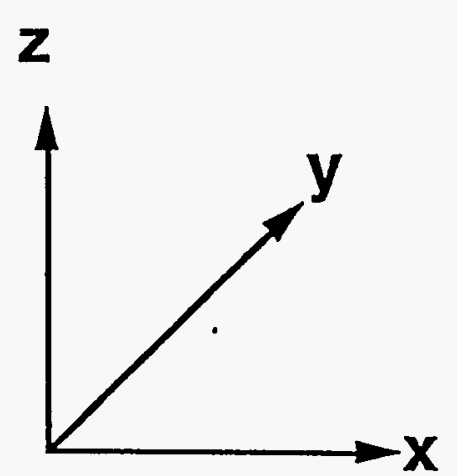

Fig. I 


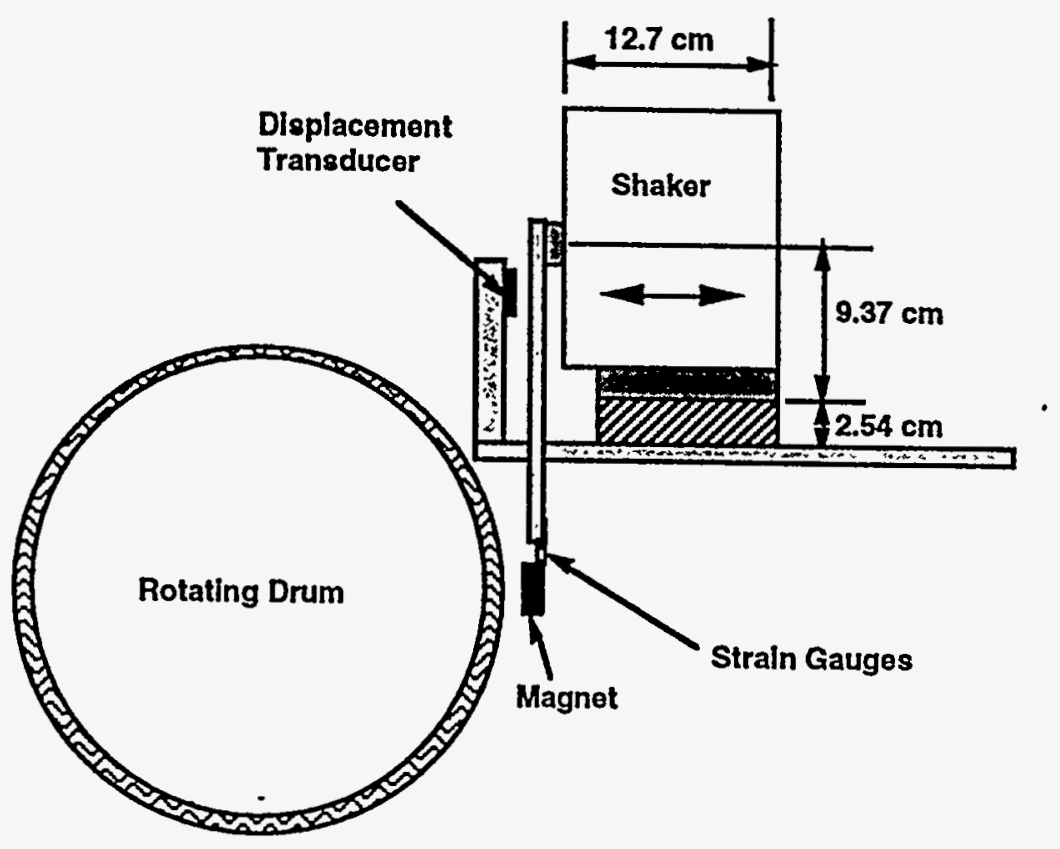




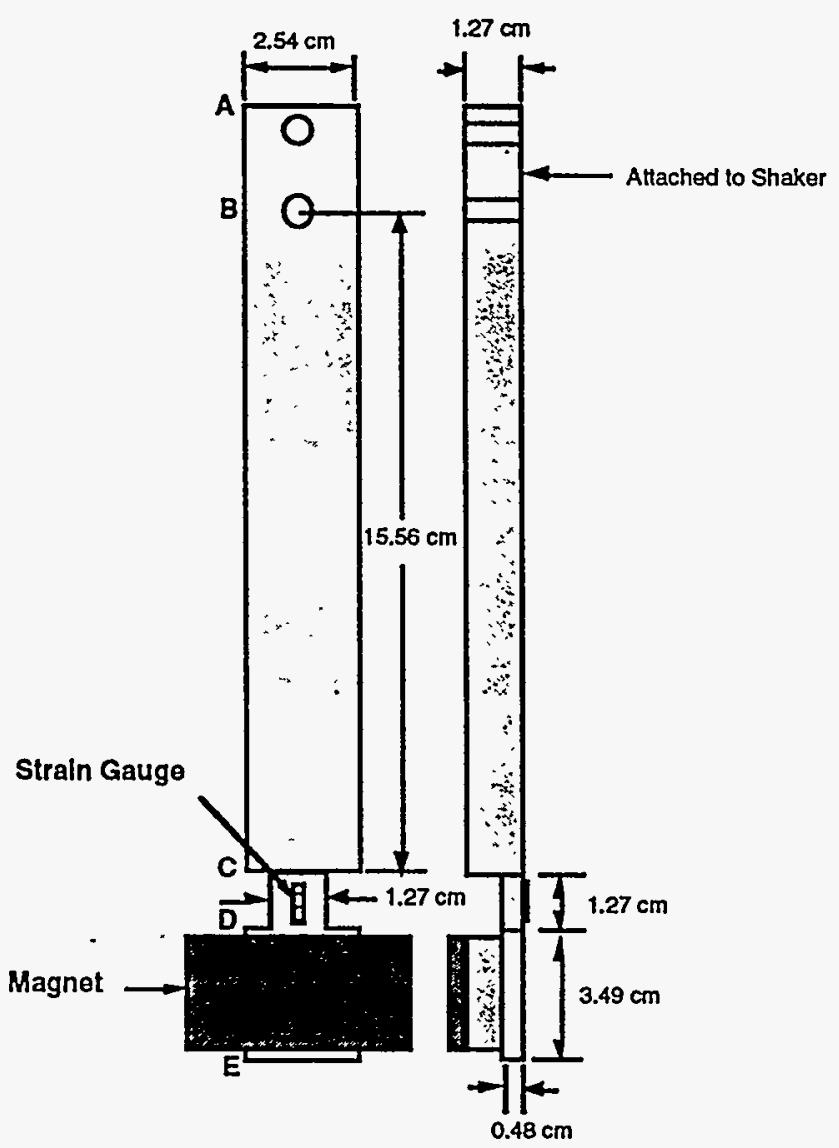

(a)

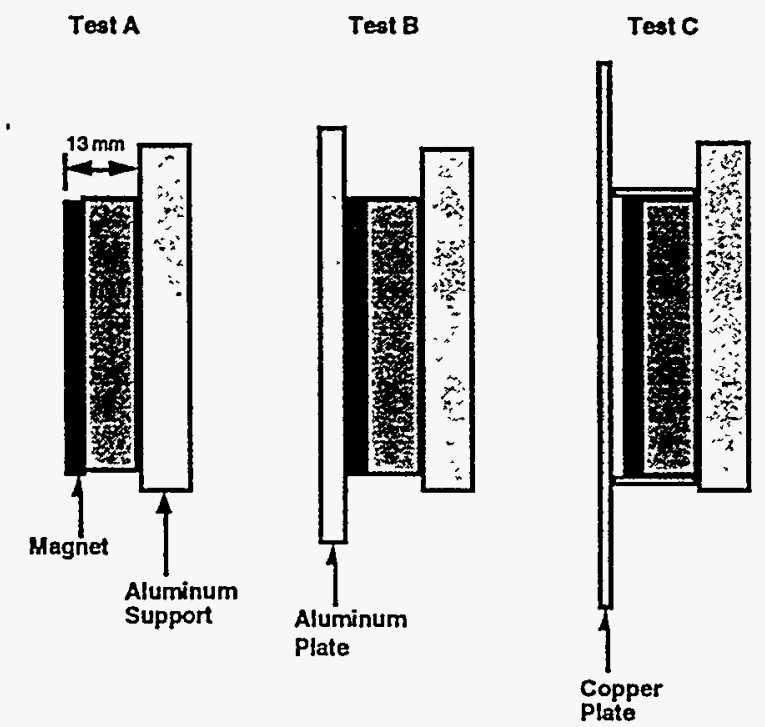

(b) 

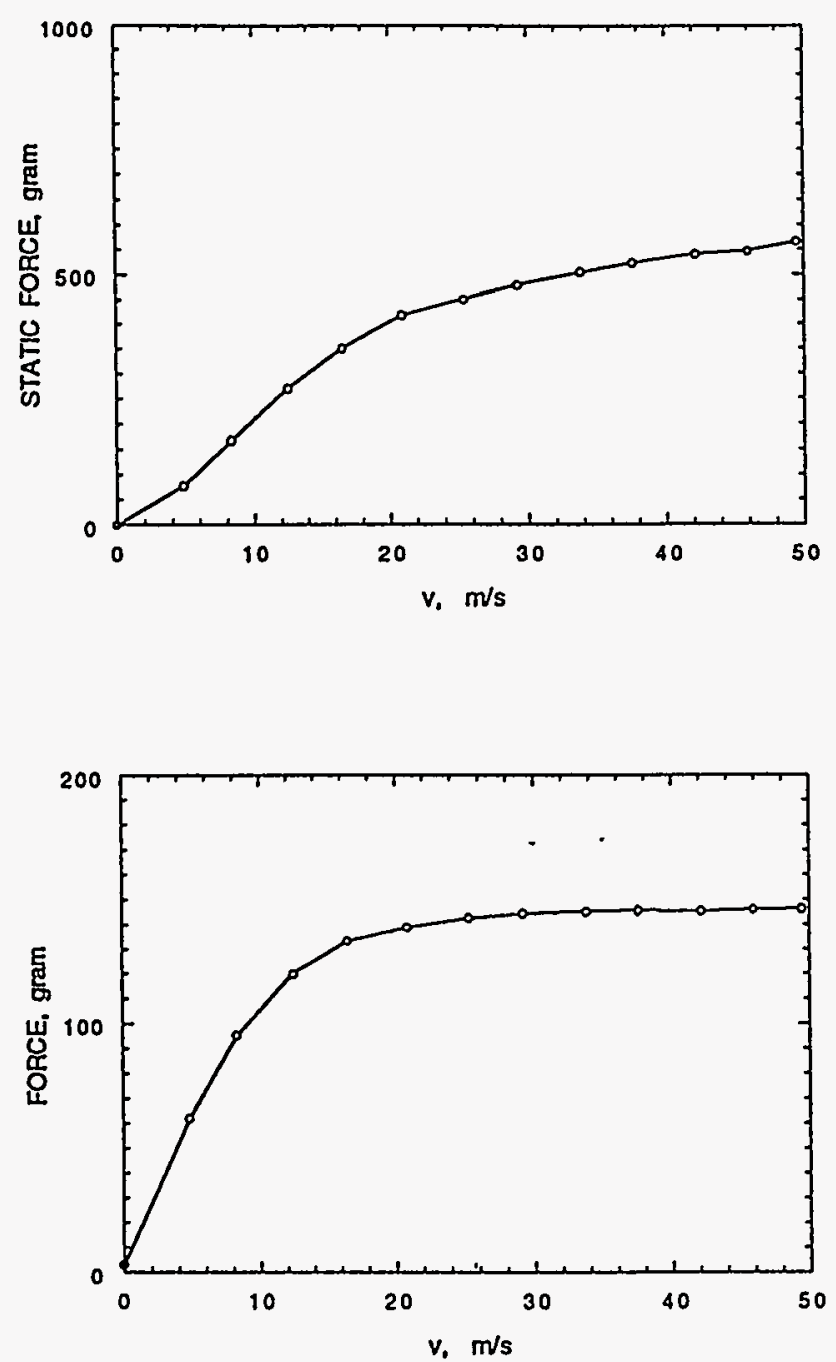
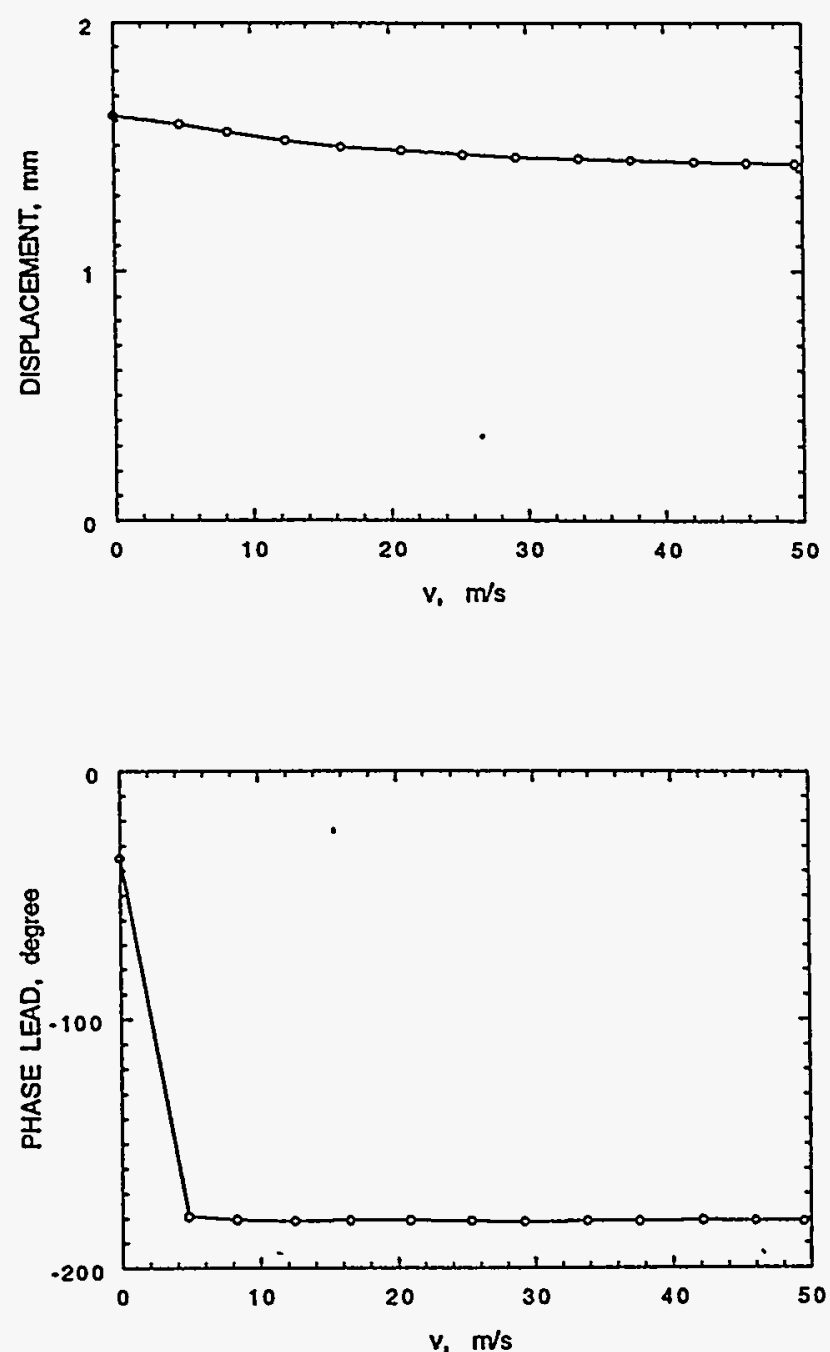

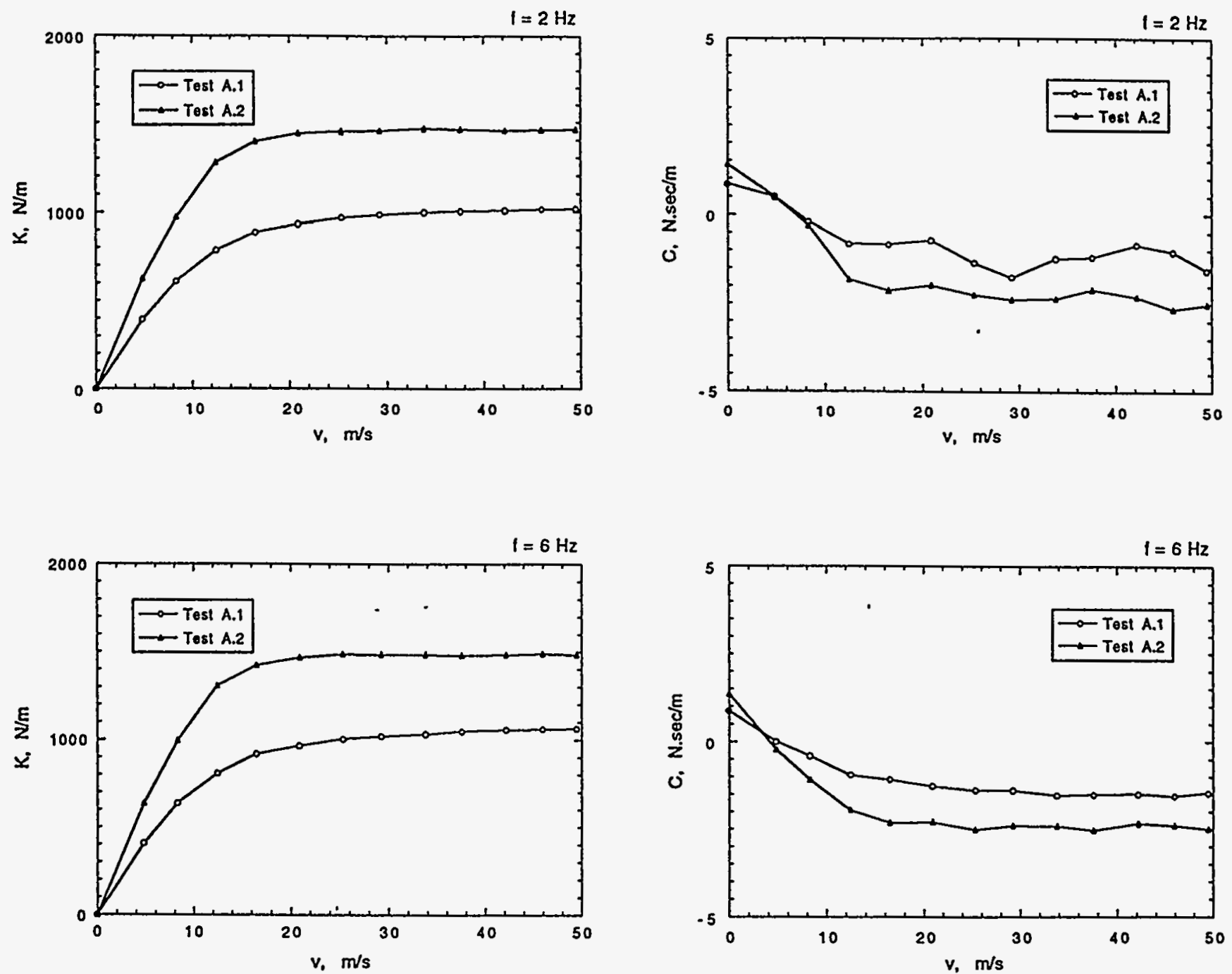

Fig. 5 

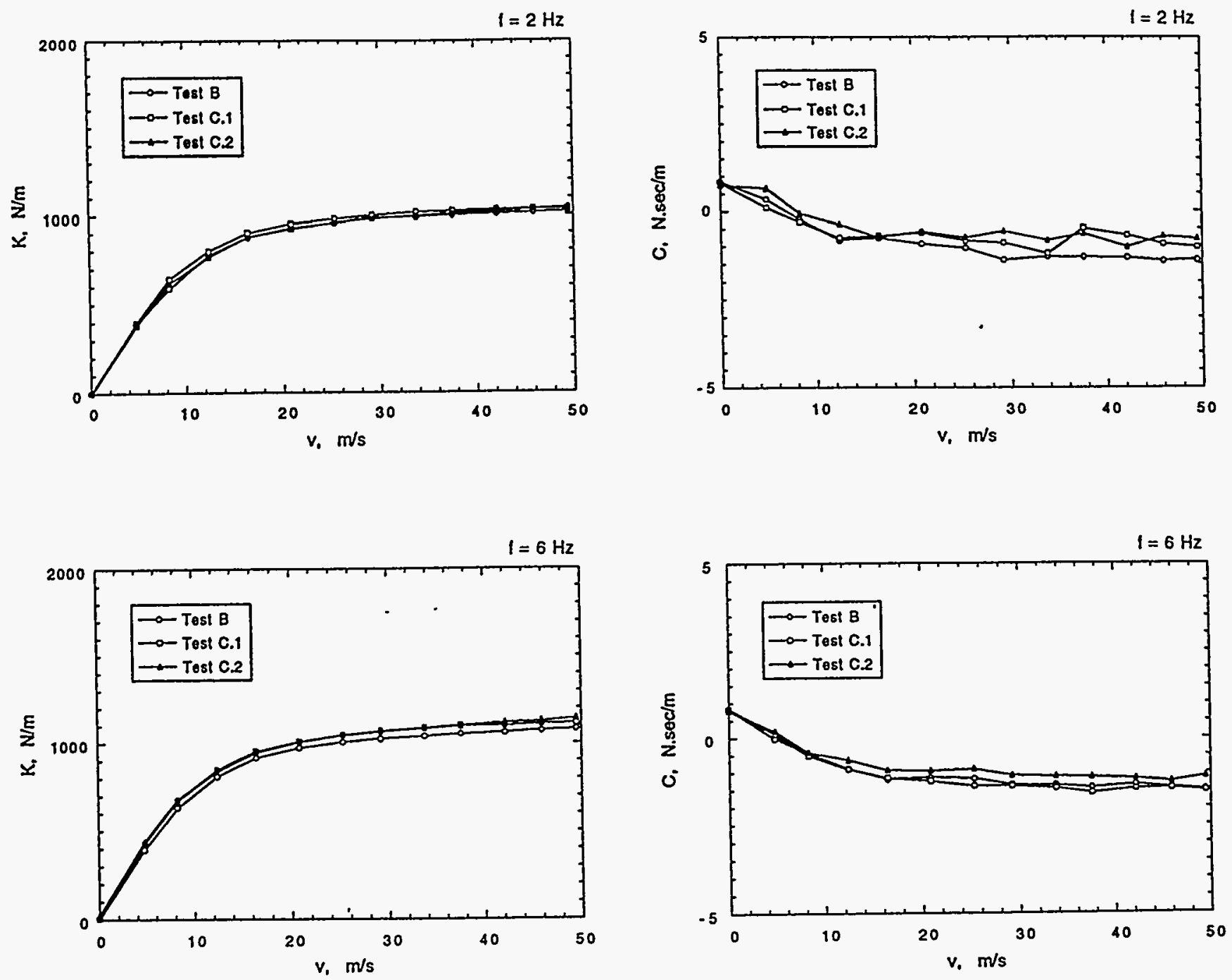


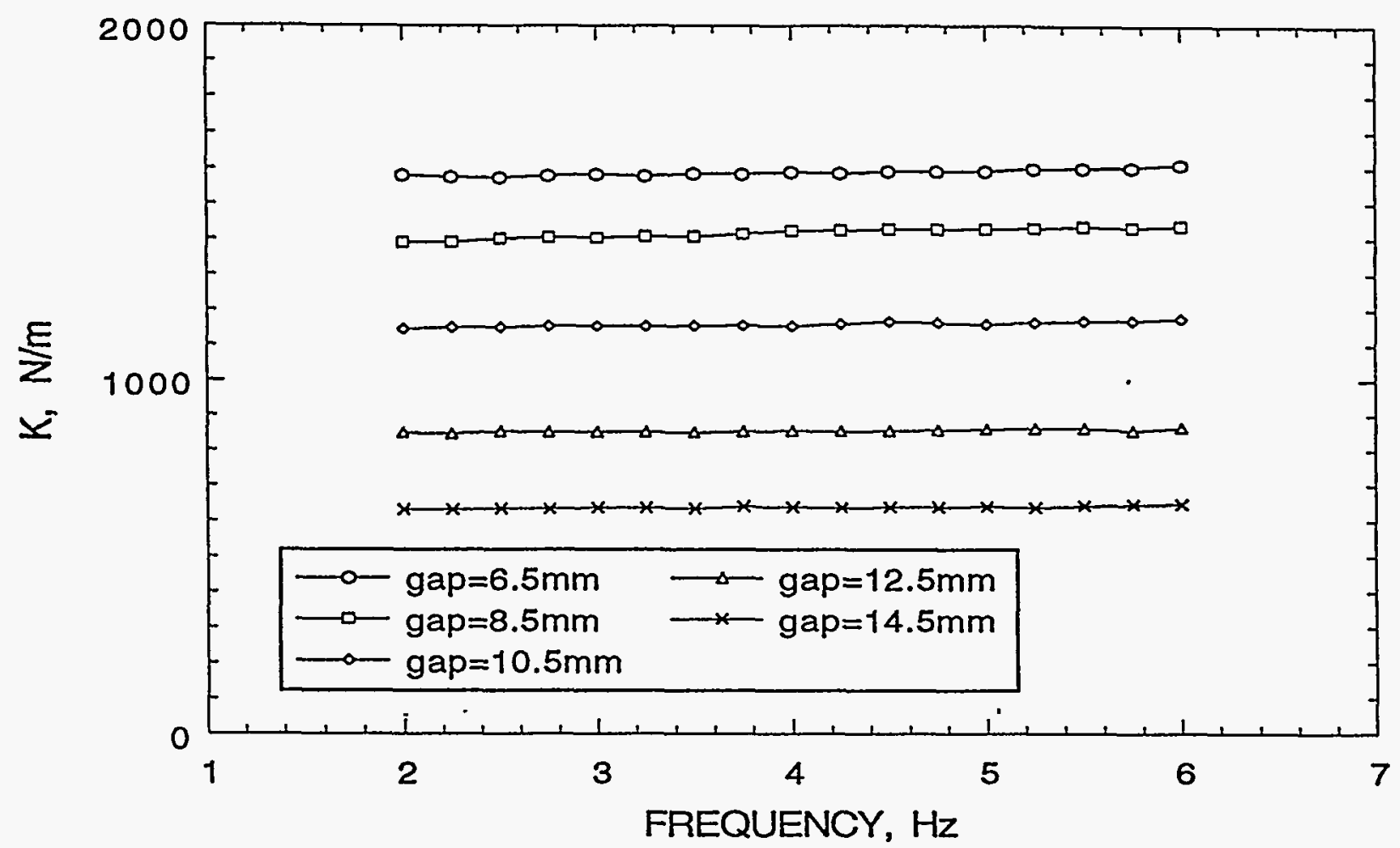

Fig. 7a 


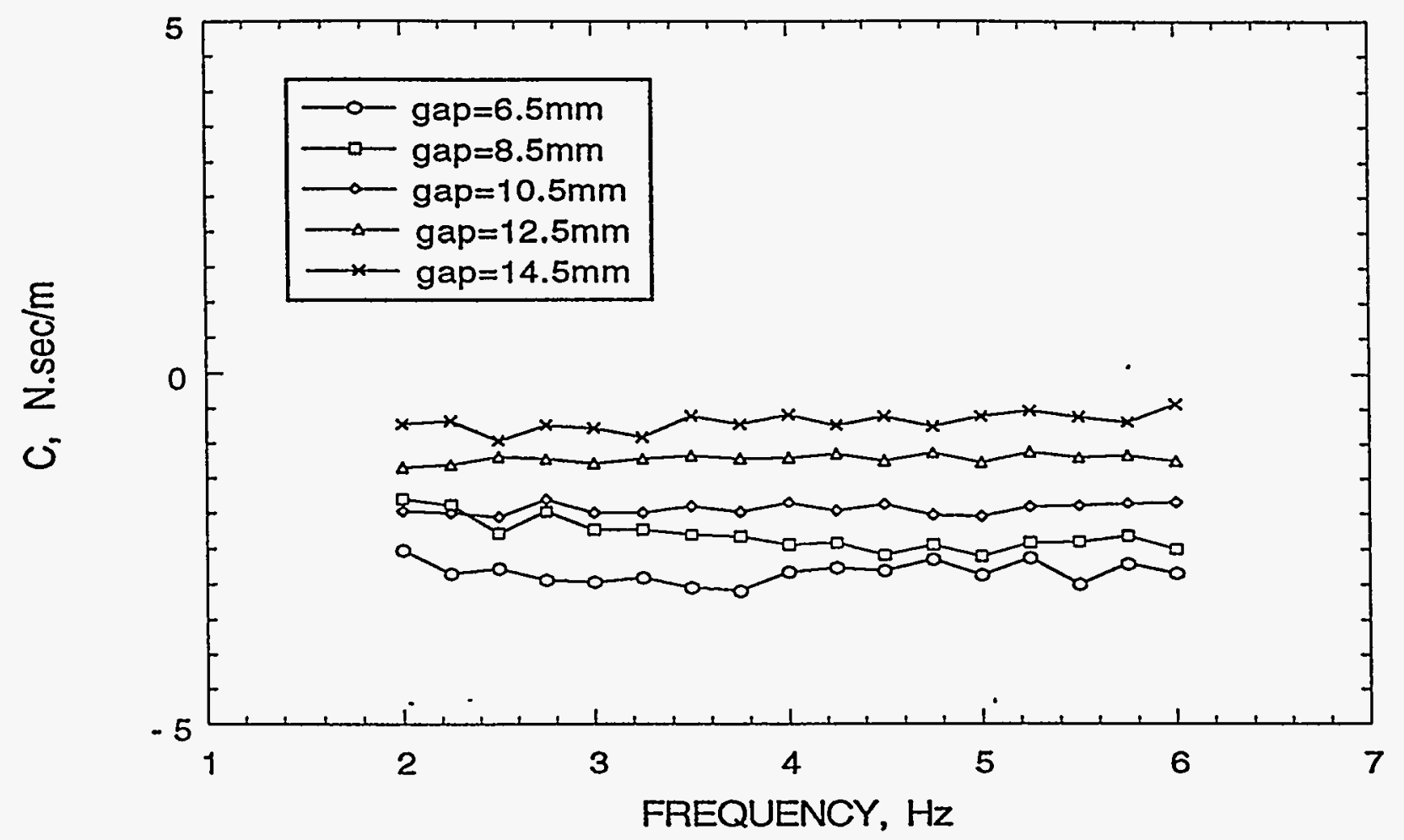

Fig. $7 b$ 

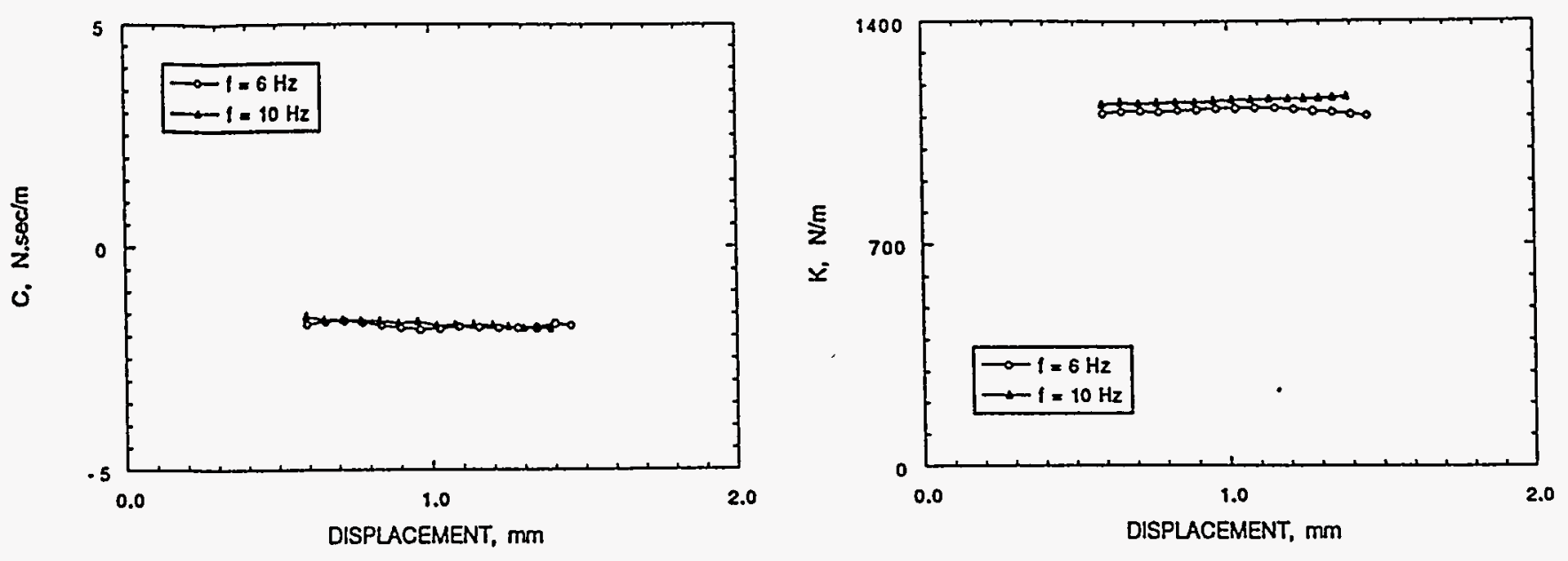
Magnet - Dummy, $v=50 \mathrm{~m} / \mathrm{s}$

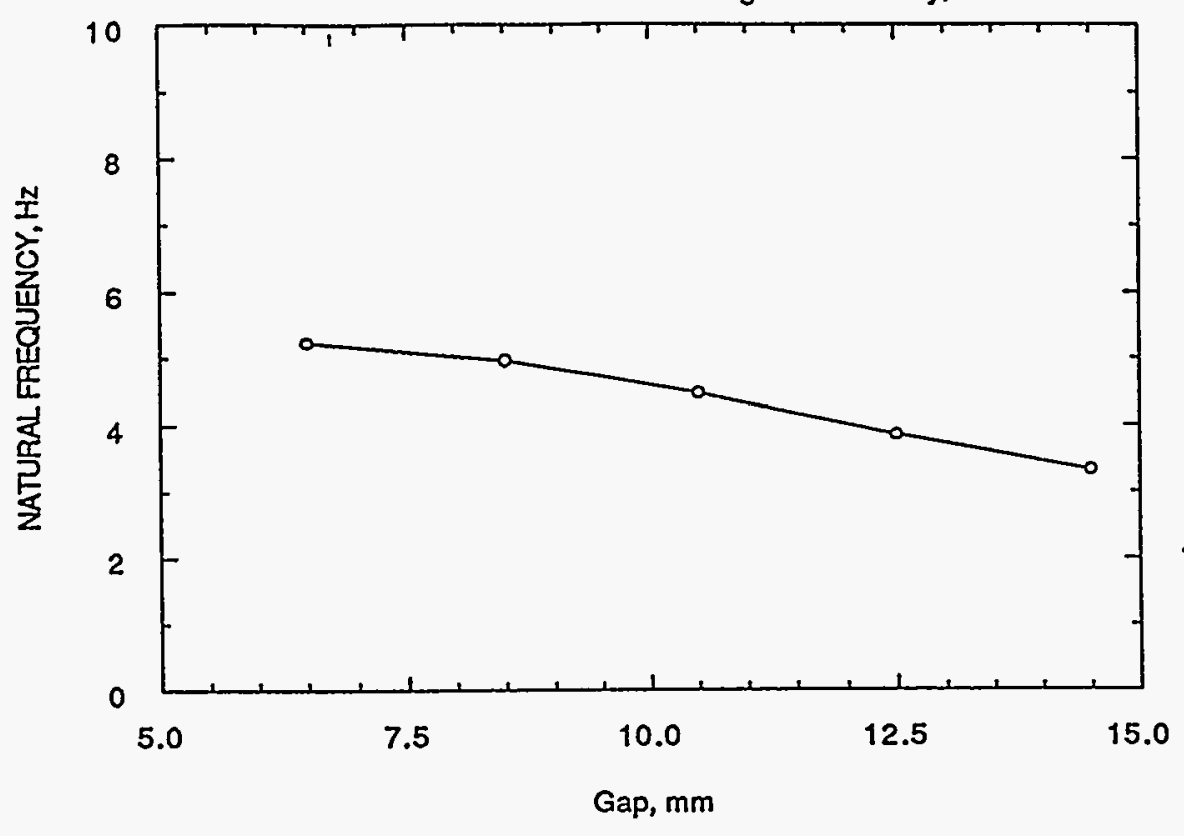

Magnet - Dummy, $v=50 \mathrm{~m} / \mathrm{s}$

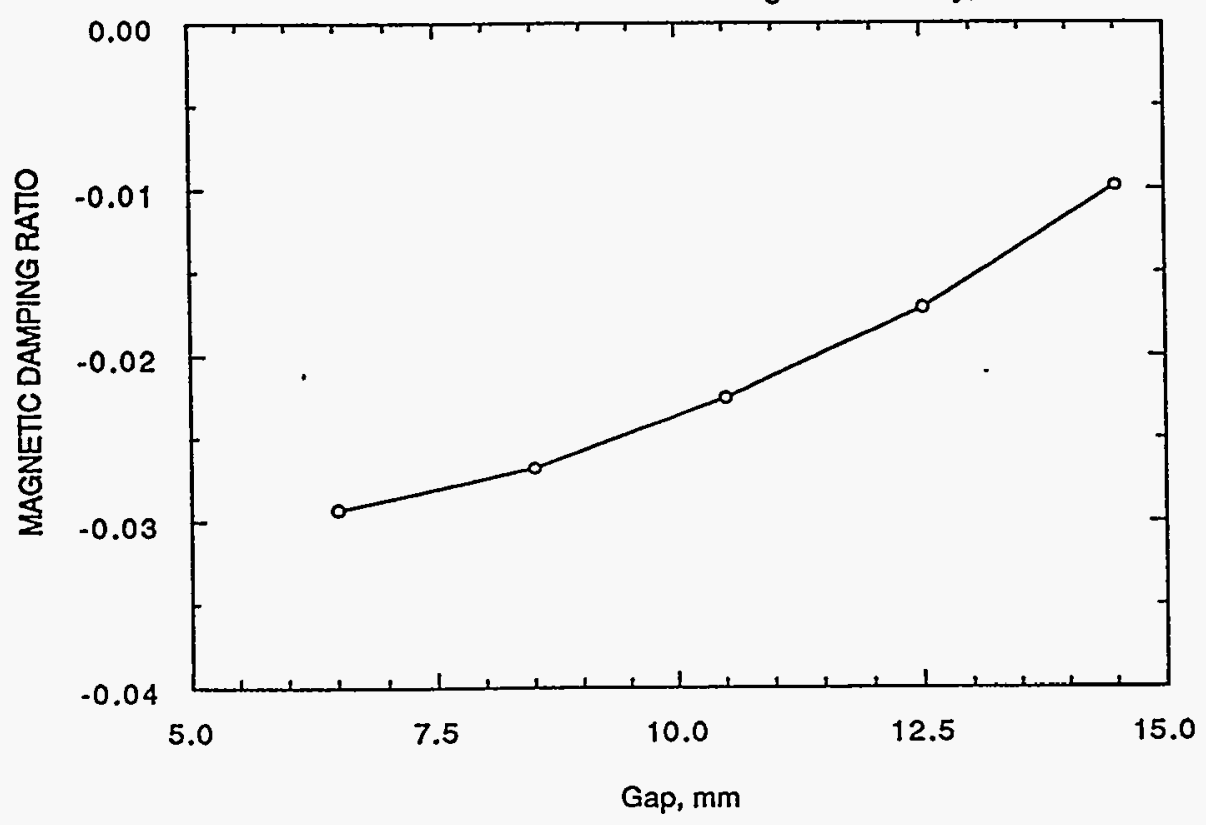

